\title{
Centroids of Lie Supertriple Systems
}

\author{
Jianrong Peng, Liangyun Chen, and Bing Sun \\ School of Mathematics and Statistics, Northeast Normal University, Changchun 130024, China \\ Correspondence should be addressed to Liangyun Chen; chenly640@nenu.edu.cn
}

Received 9 February 2015; Revised 4 June 2015; Accepted 8 June 2015

Academic Editor: Yao-Zhong Zhang

Copyright (C) 2015 Jianrong Peng et al. This is an open access article distributed under the Creative Commons Attribution License, which permits unrestricted use, distribution, and reproduction in any medium, provided the original work is properly cited.

We derive certain structural results concerning centroids of Lie supertriple systems. Centroids of the tensor product of a Lie supertriple system and a unital commutative associative algebra are studied. Furthermore, the centroid of a tensor product of a simple Lie supertriple system and a polynomial ring is partly determined.

\section{Introduction}

The notion of Lie triple systems already appeared in Cartan's work on Riemannian geometry, albeit the formal concept was not defined until 1949 by Jacobson in his study of associative algebras that are closed with respect to triple commutators (cf. [1]). The role played by Lie triple systems in the theory of symmetric spaces is parallel to that of Lie algebras in the theory of Lie groups: the tangent space at every point of a symmetric space has the structure of a Lie triple system.

As a natural generalization of Lie triple systems, the concept of Lie supertriple systems was introduced in the study of Yang-Baxter equations (cf. [2]). Lie supertriple systems have proved to be of interest in ternary structures appearing in various models in high energy physics, and many important results on Lie supertriple systems have been obtained (cf. [2-7]). In particular, the authors applied Lie supertriple systems to obtain some new solutions of the YangBaxter equation (cf. [2]).

The centroid $\Gamma(A)$ of an algebra $A$ is the set of all linear transformations $T \in \operatorname{End}(A)$ such that $T(x y)=$ $T(x) y=x T(y)$ for all $x, y \in A . \Gamma(A)$ is always a unital associative algebra, and if $A$ is "nice" (e.g., unital, prime, or simple), then $\Gamma(A)$ is a commutative ring of scalars. Centroids are interesting subjects in mathematics, and there has been a great deal of work concerning them. Benkart and Neher studied centroids of Lie algebras in [8] and Melville investigated centroids of nilpotent Lie algebras in [9]. It turns out that results on the centroids of Lie algebras are a key ingredient in the classification of extended affine Lie algebras. The centroids of Lie triple systems were mentioned by Benito et al. in [10]. Results on centroids of Lie triple system, $n$-Lie algebras, and Lie triple algebras were developed in [11-13].

In this paper we present new results concerning the centroids of Lie supertriple systems and give some conclusions of the tensor product of a Lie supertriple system and a unital commutative associative algebra. Furthermore, we completely determine the centroid of the tensor product of a simple Lie supertriple system and a polynomial ring. The paper is organized as follows. In Section 2, we list some notions of rings and Lie supertriple systems. In Section 3, we introduce the notion of centroids of Lie supertriple systems. Then we show some properties of centroids of Lie supertriple systems. In Section 4, we investigate the centroid of the tensor product of a Lie supertriple system $T$ and a unital commutative associative algebra $A$. As an important theorem in this section, we prove that, for a tensor product $\widetilde{T}=T \otimes R$ of a simple Lie supertriple system $T$ and a polynomial ring $R=\mathbf{F}\left[x_{1}, \ldots, x_{n}\right]$, its centroid is $\Gamma(\widetilde{T})_{\overline{0}}=\Gamma(T)_{\overline{0}} \otimes R$.

\section{Preliminaries}

Throughout this paper, the base field $\mathbf{F}$ is assumed to be of arbitrary characteristic unless otherwise stated.

Definition 1 (see [14]). An integral domain is a ring with no left or right zero divisors. 
Definition 2 (see [14]). An algebraically closed field $\mathbf{F}$ is a field such that every $f(x) \in \mathbf{F}[x]$ is a product of linear polynomials in $\mathbf{F}[x]$.

Definition 3 (see [15]). An idempotent of a ring is an element $a$ such that $a a=a$.

Definition 4 (see [2]). A Lie supertriple system $T$ is a $\mathbf{Z}_{2}$ graded vector space with a ternary product [, , ] satisfying the following conditions:

(1) $d([x, y, z]) \equiv d(x)+d(y)+d(z)(\bmod 2)$,

(2) $[x, y, z]=-(-1)^{d(x) d(y)}[y, x, z]$,

(3) $(-1)^{d(x) d(z)}[x, y, z]+(-1)^{d(x) d(y)}[y, z, x]+$ $(-1)^{d(y) d(z)}[z, x, y]=0$,

(4) $[u, v,[x, y, z]]=[[u, v, x], y, z]+(-1)^{(d(u)+d(v)) d(x)}[x$, $[u, v, y], z]+(-1)^{(d(u)+d(v))(d(x)+d(y))}[x, y,[u, v, z]]$,

where $x, y, z, u, v$ are homogeneous elements in $T$. We denote by $d(x)$ the $\mathbf{Z}_{2}$-graded degree of $x, \forall x \in T_{\alpha}, \alpha \in \mathbf{Z}_{2}$. In the sequel, whenever the notation $d(x)$ appears, it implies that $x$ is a homogeneous element of degree $d(x)$ in $T$.

Definition 5 (see [16]). Let $L=L_{\overline{0}} \oplus L_{\overline{1}}$ be a superalgebra whose multiplication is denoted by [, ]. This implies that $\left[L_{\alpha}, L_{\beta}\right] \subseteq L_{\alpha+\beta}$ for all $\alpha, \beta \in \mathbf{Z}_{2}$. L is a Lie superalgebra if the multiplication satisfies the following identities:

(1) $[a, b]=-(-1)^{\alpha \beta}[b, a]$;

(2) $[a,[b, c]]=[[a, b], c]+(-1)^{\alpha \beta}[b,[a, c]]$,

for any $a \in L_{\alpha}, b \in L_{\beta}, c \in L, \alpha, \beta \in \mathbf{Z}_{2}$.

Remark 6. (1) Let $T$ be a Lie superalgebra. It is clear that if we introduce a triple product $[x, y, z]$ by $[x, y, z]=[[x, y], z]$ for any $x, y, z \in T$, then $T$ becomes a Lie supertriple system. So in this sense every Lie superalgebra deduces a Lie supertriple system.

(2) Any Lie supertriple system $T$ can be considered as a subspace of a Lie superalgebra. In fact, set $L_{s}(T)$ to be the vector space $L_{s}(T)=\operatorname{InnDer}(T) \oplus T$ with $\operatorname{InnDer}(T)=$ $\{D \mid D=\Sigma L(x, y)\}$, where $L(x, y) z=[x, y, z]$, for any $x, y \in T$. The product in $L_{s}(T)$ is $\left[x_{1}+D_{1}, x_{2}+D_{2}\right]=$ $\left(L\left(x_{1}, x_{2}\right)+\left[D_{1}, D_{2}\right]\right)+\left(D_{1}\left(x_{2}\right)-(-1)^{d\left(D_{2}\right) d\left(x_{1}\right)} D_{2}\left(x_{1}\right)\right)$ for any $x_{1}, x_{2} \in T, D_{1}, D_{2} \in \operatorname{InnDer}(T)$. It is easy to see that $L_{s}(T)$ is a Lie superalgebra.

Definition 7 (see [5]). A derivation of a Lie supertriple system $T$ is a homogeneous linear map $D: T \rightarrow T$ such that

$$
\begin{aligned}
D([x, y, z])= & {[D(x), y, z] } \\
& +(-1)^{d(D) d(x)}[x, D(y), z] \\
& +(-1)^{d(D)(d(x)+d(y))}[x, y, D(z)],
\end{aligned}
$$

where $x, y, z \in T$. We denote by $d(f)$ the $\mathbf{Z}_{2}$-graded degree of a homogeneous linear mapping $f$ of $T$.
Definition 8. An ideal of a Lie supertriple system $T$ is a $\mathbf{Z}_{2}$ graded subspace $I$ for which $[I, T, T] \subseteq I$. Moreover, if $[T, I, I]=0$, then $I$ is called an abelian ideal of $T$.

Definition 9. Let $I$ be a nonempty subset of $T$. One calls $C_{T}(I)=\{x \in T \mid L(x, a)=R(a, x)=0, \forall a \in I\}$ the centralizer of $I$ in $T$, where $R(a, x) z=(-1)^{d(z)(d(a)+d(x))}[z, a, x]$. In particular, $C_{T}(T)=\{x \in T \mid[x, T, T]=0\}$ is the center of $T$, denoted by $C(T)$.

\section{Centroids of Lie Supertriple Systems}

Definition 10. Let $T$ be a Lie supertriple system over a field $\mathbf{F}$. The centroid of $T$ is the space of $\mathbf{F}$-linear transformations on $T$ given by $\Gamma(T)=\left\{\phi \in \operatorname{End}_{\mathbf{F}}(T) \mid \phi([x, y, z])=[\phi(x), y, z]\right.$, $\forall x, y, z \in T\}$.

By the definition of Lie supertriple system we conclude that if $\phi \in \Gamma(T)$ and $x, y, z \in T$, then

$$
\begin{aligned}
\phi([x, y, z]) & =[\phi(x), y, z]=(-1)^{d(\phi) d(x)}[x, \phi(y), z] \\
& =(-1)^{d(\phi)(d(x)+d(y))}[x, y, \phi(z)] .
\end{aligned}
$$

It is clear that the scalar maps will always be in the centroid.

Definition 11. Let $\phi \in \operatorname{End}_{\mathrm{F}}(T)$. If $\phi(T) \subseteq C(T)$ and $\phi([T, T, T])=0$, then $\phi$ is called a central derivation. The set of all central derivations of $T$ is denoted by $\mathrm{ZDer}(T)$.

Proposition 12. $Z \operatorname{Der}(T)$ is an ideal of $\Gamma(T)$.

Proof. It is easily seen that $\operatorname{ZDer}(T) \subseteq \Gamma(T)$. For all $\phi \epsilon$ $\mathrm{ZDer}(T)$ and $\psi \in \Gamma(T)$, we have

$$
\begin{aligned}
& {[[\phi, \psi](x), T, T]} \\
& \quad=\left[\phi \psi(x)-(-1)^{d(\phi) d(\psi)} \psi \phi(x), T, T\right] \\
& \quad=[\phi \psi(x), T, T]-(-1)^{d(\phi) d(\psi)}[\psi \phi(x), T, T]=0, \\
& {[\phi, \psi]([T, T, T])} \\
& \quad=\phi \psi([T, T, T])-(-1)^{d(\phi) d(\psi)} \psi \phi([T, T, T]) \\
& \quad=\phi([\psi(T), T, T])-(-1)^{d(\phi) d(\psi)} \psi \phi([T, T, T]) \\
& \quad=0 .
\end{aligned}
$$

Hence $\operatorname{ZDer}(T)$ is an ideal of $\Gamma(T)$.

Proposition 13. InnDer $(T)$ is the subalgebra of $\operatorname{End}_{\mathrm{F}}(T)$ and $\Gamma(T)$ is the centralizer of InnDer $(T)$. 
Proof. For any $\phi \in \Gamma(T)$ and $x, y, z \in T$, we have

$$
\begin{aligned}
{[\phi, L(x, y)](z)=} & \phi L(x, y)(z) \\
& -(-1)^{d(\phi) d L(x, y)} L(x, y) \phi(z) \\
= & \phi([x, y, z]) \\
& -(-1)^{d(\phi)(d(x)+d(y))} L(x, y) \phi(z) \\
= & 0,
\end{aligned}
$$

which proves the proposition.

If $T$ is perfect (i.e., $T=T^{(1)}=[T, T, T]$ ), then the centroid $\Gamma(T)$ is supercommutative. Indeed, for all $x, y, z \in T, \phi, \psi \in$ $\Gamma(T)$, we have

$$
\begin{aligned}
\phi \psi([x, y, z]) & =(-1)^{(d(\psi)+d(x)) d(\phi)}[\psi(x), \phi(y), z] \\
& =(-1)^{(d(\psi)+d(x)) d(\phi)} \psi[x, \phi(y), z] \\
& =(-1)^{d(\phi) d(\psi)} \psi \phi[x, y, z] .
\end{aligned}
$$

Proposition 14. If $T$ has no nonzero ideals $I, J$ with $[T, I, J]=$ 0 (i.e., $T$ is prime) and $C(T)=0$, then $\Gamma(T)_{\overline{0}}$ is an integral domain.

Proof. Clearly, id $\in \Gamma(T)_{\overline{0}}$. If there exist $\psi \neq 0$ and $\phi \neq 0$ such that $\psi \varphi=0$, then there are $x, y \in T$ such that $\psi(x) \neq 0$ and $\phi(y) \neq 0$. Then

$$
\begin{array}{r}
{[z, \psi(x), \phi(y)]} \\
=(-1)^{d(\psi) d(z)}(-1)^{(d(z)+d(x)) d(\phi)} \psi \phi([z, x, y])=0, \\
\forall z \in T .
\end{array}
$$

Since $C(T)=0,[\phi(x), T, T] \neq 0$; that is, $\phi[x, T, T] \neq$ 0 . Therefore, $\psi(x)$ generates a nonzero ideal $\psi\left(I_{x}\right)=$ $\psi[[\cdots[[x, T, T], T, T] \cdots], T, T]$. In a similar way, $\phi(y)$ generates a nonzero ideal $\psi\left(I_{y}\right)=\psi[[\cdots[[y, T, T], T, T] \cdots], T, T]$ such that $\left[T, \psi\left(I_{x}\right), \phi\left(I_{y}\right)\right]=\psi \phi\left[T, I_{x}, I_{y}\right]=0$, a contradiction. Hence $\Gamma(T)_{0}$ has no zero divisors, and so it is an integral domain.

Theorem 15. If $T$ is a simple Lie supertriple system over an algebraically closed field $\mathbf{F}$ (i.e., $T^{(1)} \neq 0$ ) and $T$ has only two ideals $T$ and 0 , then $\Gamma(T)=$ Fid.

Proof. Let $\phi \in \Gamma(T) \subseteq \operatorname{End}_{\mathbf{F}}(T)$. Since $\mathbf{F}$ is algebraically closed, $\phi$ has an eigenvalue $\lambda$. We denote the corresponding eigenspace by $E_{\lambda}(\phi)$. So $E_{\lambda}(\phi) \neq 0$. For any $x \in E_{\lambda}(\phi)$, $x=x_{0}+x_{1}$, where $x_{0} \in T_{0}, x_{1} \in T_{1}$, we have $\phi(x)=\phi\left(x_{0}\right)+$ $\phi\left(x_{1}\right)=\lambda x=\lambda x_{0}+\lambda x_{1}$, so $\phi\left(x_{0}\right)-\lambda x_{0}=\phi\left(x_{1}\right)-\lambda x_{1} \in T_{0} \cap T_{1}$; that is, $\phi\left(x_{0}\right)=\lambda x_{0}, \phi\left(x_{1}\right)=\lambda x_{1}$. So we have $x_{0} \in T_{0}$ and $x_{1} \in T_{1}$. Therefore $E_{\lambda}(\phi)$ is a $\mathbf{Z}_{2}$-graded vector space. For any $v \in E_{\lambda}(\phi), x, y \in T$, we have $\phi([v, x, y])=[\phi(v), x, y]=$ $\lambda[v, x, y]$, so $[v, x, y] \in E_{\lambda}(\phi)$. It follows that $E_{\lambda}(\phi)$ is an ideal of $T$. But $T$ is simple, so $E_{\lambda}(\phi)=T$; that is, $\phi=\lambda \mathrm{id}_{T}$. This proves the theorem.
When $\Gamma(T)=$ Fid, the Lie supertriple system $T$ is said to be central. Furthermore, if $T$ is simple, $T$ is said to be central simple. Every simple Lie supertriple system is central simple over its centroid.

Proposition 16. Let $T$ be a Lie supertriple system over a field F. Then

(1) $T$ is indecomposable (cannot be written as the direct sum of two nontrivial ideals) if and only if $\Gamma(T)$ does not contain idempotents except 0 and id;

(2) if $T$ is perfect, every $\phi \in \Gamma(T)$ is supersymmetric with respect to any invariant form on $T$.

Proof. Consider the following:

(1) If $\phi \in \Gamma(T)$ is an idempotent and satisfies $\phi \neq 0$, id, then $\phi^{2}(x)=\phi(x), \forall x \in T$. We can see that $\operatorname{Ker} \phi$ and $\operatorname{Im} \phi$ are ideals of $T$. In fact, for any $x \in \operatorname{Ker} \phi$ and $y, z \in T$, we have $x=x_{0}+x_{1}$, where $x_{0} \in T_{0}$ and $x_{1} \in T_{1}$; then $\phi\left(x_{0}\right) \in T_{0}$, $\phi\left(x_{1}\right) \in T_{1}$ and $\phi(x)=\phi\left(x_{0}\right)+\phi\left(x_{1}\right)=0, \phi\left(x_{0}\right)=\phi\left(x_{1}\right)=0$, so $x_{0} \in \operatorname{Ker} \phi$ and $x_{1} \in \operatorname{Ker} \phi$; that is, $\operatorname{Ker} \phi$ is a $\mathbf{Z}_{2}$-graded vector space. $\phi([x, y, z])=[\phi(x), y, z]=0$ implies $[x, y, z] \epsilon$ Ker $\phi$. For any $x \in \operatorname{Im} \phi$, we have $x=x_{0}+x_{1}$, where $x_{0} \in T_{0}$ and $x_{1} \in T_{1}$; there exists $a \in T$ such that $x=\phi(a), a=a_{0}+a_{1}$, $a_{0} \in T_{0}, a_{1} \in T_{1}$, so $x=\phi\left(a_{0}\right)+\phi\left(a_{1}\right)$; that is, $x_{0}-\phi\left(a_{0}\right)=$ $x_{1}-\phi\left(a_{1}\right) \in T_{0} \cap T_{1}$; then $x_{0}=\phi\left(a_{0}\right)$ and $x_{1}=\phi\left(a_{1}\right)$; that is, $\operatorname{Im} \phi$ is a $\mathbf{Z}_{2}$-graded vector space. For any $y, z \in \operatorname{Im} \phi$, we have $[x, y, z]=[\phi(a), y, z]=\phi([a, y, z]) \in \operatorname{Im} \phi, \forall y, z \in T$. Moreover, $\operatorname{Ker} \phi \cap \operatorname{Im} \phi=0$. Indeed, if $x \in \operatorname{Ker} \phi \cap \operatorname{Im} \phi$, then there exists $y \in T$ such that $x=\phi(y)$ and $0=\phi(x)=\phi^{2}(y)=$ $\phi(y)=x$. We have a decomposition $x=\phi(x)+y, \forall x \in T$, where $\phi(y)=0$. So we have $T=\operatorname{Ker} \phi \oplus \operatorname{Im} \phi$, a contradiction.

On the other hand, suppose $T$ has a decomposition $T=$ $T_{1} \oplus T_{2}$. Then for any $x \in T$, we have $x=x_{1}+x_{2}, x_{i} \in T_{i}, i=$ 1,2 . We choose $\phi \in \Gamma(T)$ such that $\phi\left(x_{1}\right)=x_{1}$ and $\phi\left(x_{2}\right)=0$. Then $\phi^{2}(x)=\phi\left(x_{1}\right)=x_{1}=\phi(x)$. Hence $\phi$ is an idempotent. By assumption, we know $\phi=0$ or $\phi=$ id. If $\phi=0$, then $x_{1}=0$ implying $T=T_{2}$. If $\phi=\mathrm{id}$, then $x_{2}=0$ implying $T=T_{1}$.

(2) Let $f$ be an invariant bilinear form on $T$. Then

$$
\begin{array}{r}
f([x, y, u], v) \\
=(-1)^{d(u) d(v)+d(u) d(y)+d(v) d(y)} f(x,[v, u, y]), \\
\forall x, y, u, v \in T .
\end{array}
$$

Since $T$ is perfect, for $\phi \in \Gamma(T)$, we have

$$
\begin{aligned}
f & (\phi([x, y, u]), v)=(-1)^{d(x) d(\phi)} f([x, \phi(y), u], v) \\
& =(-1)^{d(x) d(\phi)}(-1)^{(d(\phi)+d(y)) d(v)+(d(\phi)+d(y)) d(u)+d(v) d(u)} \\
& \cdot f(x,[v, u, \phi(y)]) \\
& =(-1)^{d(x) d(\phi)+(d(\phi)+d(y)) d(v)+(d(\phi)+d(y)) d(u)+d(v) d(u)} \\
& \cdot(-1)^{d(\phi)(d(u)+d(v))} f([x, \phi([v, u, y])]) \\
& =(-1)^{d(x) d(\phi)+(d(\phi)+d(y)) d(v)+(d(\phi)+d(y)) d(u)+d(v) d(u)}
\end{aligned}
$$




$$
\begin{aligned}
& \cdot(-1)^{d(\phi)(d(u)+d(v))} f(x,[\phi(v), u, y]) \\
& =(-1)^{d(x) d(\phi)+(d(\phi)+d(y)) d(v)+(d(\phi)+d(y)) d(u)+d(v) d(u)} \\
& \cdot(-1)^{d(\phi)(d(u)+d(v))} \\
& \cdot(-1)^{(d(\phi)+d(v)) d(u)+(d(\phi)+d(v)) d(y)+d(y) d(u)} \\
& \cdot f([x, y, u], \phi(v))=(-1)^{d(\phi)(d(x)+d(y)+d(u))} \\
& \cdot f([x, y, u], \phi(v)),
\end{aligned}
$$

which proves the proposition.

Proposition 17. Let $T$ be a Lie supertriple system over a field $\mathbf{F}$ and $I$ a subset of $T$. Then $C_{T}(I)$ is invariant under $\Gamma(T)$ and so is any perfect ideal of $T$.

Proof. For any $\phi \in \Gamma(T), x \in C_{T}, y \in I$, and $z \in T$, we have

$$
\begin{aligned}
& L(\phi(x), y)(z)=[\phi(x), y, z]=\phi([x, y, z])=0, \\
& R(y, \phi(x))(z)=(-1)^{d(z)(d(\phi)+d(x)+d(y))}[z, y, \phi(x)] \\
& \quad=(-1)^{d(z)(d(\phi)+d(x)+d(y))}(-1)^{d(\phi)(d(z)+d(y))} \phi([z, y, x]) \\
& \quad=0
\end{aligned}
$$

which implies that $\phi(x) \in C_{T}(I)$. So $C_{T}(I)$ is invariant under $\Gamma(T)$.

Let $J$ be any perfect ideal of $T$; then $J=[J, J, J]$. For any $y \in J$, there exist $a, b, c \in J$ such that $y=[a, b, c]$ and then we have $\phi(y)=\phi([a, b, c])=(-1)^{d(a) d(\phi)}[a, \phi(b), c] \in[J, T, T] \subseteq$ $J$. Hence $J$ is invariant under $\Gamma(T)$.

Theorem 18. Let $\pi: T_{1} \rightarrow T_{2}$ be an epimorphism of Lie supertriple systems. For any $f \in \operatorname{End}_{\mathbf{F}}\left(T_{1} ; \operatorname{Ker} \pi\right):=\{g \in$ $\left.\operatorname{End}_{\mathbf{F}}\left(T_{2}\right) \mid g(\operatorname{Ker} \pi) \subseteq \operatorname{Ker} \pi\right\}$ there exists a unique $\bar{f} \epsilon$ End $_{\mathbf{F}}\left(T_{2}\right)$ satisfying $\pi \circ f=\bar{f} \circ \pi$. Moreover, the following results hold:

(1) The map $\pi_{\text {End }}: \operatorname{End}_{\mathbf{F}}\left(T_{1} ; \operatorname{Ker} \pi\right) \rightarrow \operatorname{End}_{\mathbf{F}}\left(T_{2}\right), f \mapsto$ $\bar{f}$ is an algebra homomorphism satisfying the following properties:

$$
\begin{aligned}
\pi_{\text {End }}\left(\operatorname{Mult}\left(T_{1}\right)\right) & =\operatorname{Mult}\left(T_{2}\right), \\
\pi_{\text {End }}\left(\Gamma\left(T_{1}\right) \cap \operatorname{End}_{\mathbf{F}}\left(T_{1} ; \operatorname{Ker} \pi\right)\right) & \subseteq \Gamma\left(T_{2}\right) .
\end{aligned}
$$

By restriction, there is an algebra homomorphism

$$
\pi_{\Gamma}: \Gamma\left(T_{1}\right) \cap \operatorname{End}_{\mathbf{F}}\left(T_{1} ; \operatorname{Ker} \pi\right) \longrightarrow \Gamma\left(T_{2}\right), \quad f \longmapsto \bar{f}
$$

If $\operatorname{Ker} \pi=C\left(T_{1}\right)$, then every $\phi \in \Gamma\left(T_{1}\right)$ leaves Ker $\pi$ invariant; hence $\pi_{\Gamma}$ is defined on all of $\Gamma\left(T_{1}\right)$.

(2) Suppose $T_{1}$ is perfect and Ker $\pi \subseteq C\left(T_{1}\right)$. Then

$$
\pi_{\Gamma}: \Gamma\left(T_{1}\right) \cap \operatorname{End}_{\mathbf{F}}\left(T_{1} ; \operatorname{Ker} \pi\right) \longrightarrow \Gamma\left(T_{2}\right), \quad f \longmapsto \bar{f}
$$

is injective.
(3) If $T_{1}$ is perfect, $C\left(T_{2}\right)=0$, and $\operatorname{Ker} \pi \subseteq C\left(T_{1}\right)$, then $\pi_{\Gamma}: \Gamma\left(T_{1}\right) \rightarrow \Gamma\left(T_{2}\right)$ is an algebra monomorphism.

Proof. Consider the following:

(1) It is easy to see that $\pi_{\text {End }}$ is an algebra homomorphism. Since Ker $\pi$ is an ideal of $T_{1}$ and all left and right multiplication operators of $T_{1}$ leave $\operatorname{Ker} \pi$ invariant, $\operatorname{Mult}\left(T_{1}\right) \subseteq \operatorname{End}_{\mathrm{F}}\left(T_{1} ; \operatorname{Ker} \pi\right)$. Furthermore, for the left multiplication operator $L(x, y)$ on $T_{1}$ we have $\pi \circ L(x, y)=$ $L(\pi(x), \pi(y)) \circ \pi$, so $\pi_{\text {End }}(L(x, y))=L(\pi(x), \pi(y))$. For the right multiplication we have the analogous formula $\pi_{\text {End }}(R(x, y))=R(\pi(x), \pi(y))$. Moreover, $\pi$ is an epimorphism, so $\pi_{\text {End }}\left(\operatorname{Mult}\left(T_{1}\right)\right)=\operatorname{Mult}\left(T_{2}\right)$. Now we show that $\pi_{\text {End }}\left(\Gamma\left(T_{1}\right) \cap \operatorname{End}_{\mathrm{F}}\left(T_{1} ; \operatorname{Ker} \pi\right)\right) \subseteq \Gamma\left(T_{2}\right)$. Let $\phi \in \Gamma\left(T_{1}\right) \cap$ End $_{\mathbf{F}}\left(T_{1} ; \operatorname{Ker} \pi\right)$. For any $x^{\prime}, y^{\prime}, z^{\prime} \in T_{2}$ there exist $x, y, z \in T_{1}$ such that $\pi(x)=x^{\prime}, \pi(y)=y^{\prime}$, and $\pi(z)=z^{\prime}$. Then we have

$$
\begin{aligned}
\bar{\phi}\left(\left[x^{\prime}, y^{\prime}, z^{\prime}\right]\right) & =\bar{\phi}(\pi([x, y, z]))=\pi(\phi([x, y, z])) \\
& =\pi([x, y, \phi(z)]) \\
& =[\pi(x), \pi(y), \bar{\phi}(\pi(z))] \\
& =\left[x^{\prime}, y^{\prime}, \bar{\phi}\left(z^{\prime}\right)\right]
\end{aligned}
$$

which proves $\bar{\phi} \in \Gamma\left(T_{2}\right)$.

(2) If $\bar{\phi}=0$ for $\phi \in \Gamma\left(T_{1}\right) \cap \operatorname{End}_{\mathrm{F}}\left(T_{1} ; \operatorname{Ker} \pi\right)$, then $\pi\left(\phi\left(T_{1}\right)\right)=\bar{\phi}\left(\pi\left(T_{1}\right)\right)=0$, which means that $\phi\left(T_{1}\right) \subseteq \operatorname{Ker} \pi \subseteq$ $C\left(T_{1}\right)$. Hence $\phi([x, y, z])=[\phi(x), y, z]=0, \forall x, y, z \in T_{1}$. Furthermore, since $T_{1}=T_{1}^{(1)}$, we can get $\phi=0$.

(3) We can see that $\pi\left(C\left(T_{1}\right)\right) \subseteq C\left(T_{2}\right)=0$ and then $C\left(T_{1}\right) \subseteq \operatorname{Ker} \pi$. So Ker $\pi \subseteq C\left(T_{1}\right)$. By (1), we know that $\pi_{\Gamma}$ : $\Gamma\left(T_{1}\right) \rightarrow \Gamma\left(T_{2}\right)$ is a well-defined algebra homomorphism, which is an injection by (2).

Proposition 19. If the characteristic of $\mathbf{F}$ is not 2, then

$$
Z \operatorname{Der}(T)=\Gamma(T) \cap \operatorname{Der}(T) .
$$

Proof. If $\psi \in \Gamma(T) \cap \operatorname{Der}(T)$, then by the definitions of $\Gamma(T)$ and $\operatorname{Der}(T)$, for all $x, y, z \in T$, we have $\psi([x, y, z])=$ $[\psi(x), y, z]+(-1)^{d(\psi) d(x)}[x, \psi(y), z]+(-1)^{d(\psi)(d(x)+d(y))}[x$, $y, \psi(z)]$, and $\psi([x, y, z])=[\psi(x), y, z]=(-1)^{d(\psi) d(x)}[x$, $\psi(y), z]=(-1)^{d(\psi)(d(x)+d(y))}[x, y, \psi(z)]$, so $\psi([T, T, T])=0$ and $\psi(T) \subseteq \mathrm{C}(T)$. It follows easily that $\Gamma(T) \cap \operatorname{Der}(T) \subseteq$ $\mathrm{ZDer}(T)$

To show the inverse inclusion, let $\psi \in \operatorname{ZDer}(T)$; then $0=\psi([x, y, z])=[\psi(x), y, z]=(-1)^{d(\psi) d(x)}[x, \psi(y), z]=$ $(-1)^{d(\psi)(d(x)+d(y))}[x, y, \psi(z)]$. Thus, $\psi \in \Gamma(T) \cap \operatorname{Der}(T)$.

Lemma 20. Let $I$ be a nonzero $\Gamma(T)$-invariant ideal of $T$, $V(I)=\{\psi \in \Gamma(T) \mid \psi(I)=0\}$, and $\operatorname{Hom}\left(T / I, C_{T}(I)\right)$ the vector space of all linear maps from $T / I$ to $C_{T}(I)$ over $\mathbf{F}$. Define $T(I)=\left\{f \in \operatorname{Hom}\left(T / I, C_{T}(I)\right) \mid f([\bar{x}, \bar{y}, \bar{z}])=[f(\bar{x}), y, z]=\right.$ $(-1)^{d(f) d(x)}[x, f(\bar{y}), z]=(-1)^{d(f)(d(x)+d(y))}[x, y, f(\bar{z})]$, for $\bar{x}, \bar{y}, \bar{z} \in T / I\}$. Then

(1) $T(I)$ is a subspace of $\operatorname{Hom}\left(T / I, \mathrm{C}_{T}(I)\right)$ and $V(I) \cong$ $T(I)$ as vector spaces;

(2) if $\Gamma(I)=$ Fid $_{I}, \Gamma(T)=$ Fid $_{T} \oplus V(I)$ as vector spaces. 
Proof. (1) For all $f=f_{0}+f_{1} \in T(I), f_{0} \in \operatorname{Hom}\left(T / I, \mathrm{C}_{T}(I)\right)_{0}$, and $f_{1} \in \operatorname{Hom}\left(T / I, \mathrm{C}_{T}(I)\right)_{1}$, we have

$$
\begin{aligned}
f([\bar{x}, \bar{y}, \bar{z}]) & =\left(f_{0}+f_{1}\right)([\bar{x}, \bar{y}, \bar{z}])=[f(\bar{x}), y, z] \\
& =\left[\left(f_{0}+f_{1}\right)(\bar{x}), y, z\right] .
\end{aligned}
$$

Hence $f_{0}([\bar{x}, \bar{y}, \bar{z}])=\left[f_{0}(\bar{x}), y, z\right]$ and $f_{1}([\bar{x}, \bar{y}, \bar{z}])=$ $\left[f_{1}(\bar{x}), y, z\right]$; that is, $T(I)$ is a $\mathbf{Z}_{2}$-graded subspace of $\operatorname{Hom}\left(T / I, \mathrm{C}_{T}(I)\right)$. For all $\psi \in V(I), \psi=\psi_{0}+\psi_{1}$, where $\psi_{0} \in \Gamma(T)_{0}$ and $\psi_{1} \in \Gamma(T)_{1}$. Also, $\psi(x)=\psi_{0}(x)+\psi_{1}(x)=0$, $\forall x \in I$; then $\psi_{0}(x)=\psi_{1}(x)=0$; that is, $V(I)$ is a $\mathbf{Z}_{2}$-graded vector space. Since $\psi \phi(I)=\psi(\phi(I))=0=\phi(\psi(I))=\phi \psi(I)$, $\forall \phi \in \Gamma(T)$, we have $\psi \phi, \phi \psi \in V(I)$. So $V(I)$ is an ideal of the associative algebra $\Gamma(T)$. To prove (1) consider the following map $\alpha: V(I) \rightarrow T(I)$ given by

$$
\alpha(\psi)(\bar{y})=\psi(y),
$$

where $\psi \in V(I)$ and $\bar{y}=y+I \in T / I$. The map $\alpha$ is well defined. If $\bar{y}=\bar{y}_{1}$, then $y-y_{1} \in I$, and so $\psi\left(y-y_{1}\right)=0$. It follows easily that $\alpha$ is injective. We now show that $\alpha$ is onto. For every $f \in T(I)$, set $\psi_{f}: T \rightarrow T, \psi_{f}(x)=f(\bar{x})$, for all $x \in T$. It follows from the definition of $T(I)$ that, for all $x, y, z \in T, \psi_{f}([x, y, z])=f([\bar{x}, \bar{y}, \bar{z}])=[f(\bar{x}), y, z]=$ $(-1)^{d(f) d(x)}[x, f(\bar{y}), z]=(-1)^{d(f)(d(x)+d(y))}[x, y, f(\bar{z})]$. Thus $\psi_{f} \in \Gamma(T)$, and so $\psi_{f} \in V(I), \psi_{f}(I)=0$. But $\alpha\left(\psi_{f}\right)=$ $f$ implies that $\alpha$ is onto. It is easy to see that $\alpha$ preserves operations on vector spaces $T / I$ to $C_{T}(I)$. This proves (1).

We now prove (2). If $\Gamma(I)=$ Fid $_{I}$, then, for all $\psi \in$ $\Gamma(T),\left.\psi\right|_{I}=\lambda \mathrm{id}_{I}$, for some $\lambda \in \mathbf{F}$. If $\psi \neq \lambda \mathrm{id}_{T}$, let $\varphi(x)=\lambda x$, for all $x \in T$; then $\psi \in \Gamma(T)$ and $\psi-\varphi \in V(I)$. Clearly, $\psi=\varphi+(\psi-\varphi)$. Furthermore, Fid $_{T} \cap V(I)=0$, and so (2) is proved.

Lemma 21. Let $T$ be a Lie supertriple system; then $\varphi D$ is a derivation for $\varphi \in \Gamma(T)$ and $D \in \operatorname{Der}(T)$.

Proof. If $x, y, z \in T$, then

$$
\begin{aligned}
\varphi D & ([x, y, z])=[D(x), y, z]+(-1)^{d(D) d(x)} \\
& \cdot[x, D(y), z]+(-1)^{d(D)(d(x)+d(y))}[x, y, D(z)] \\
& =\varphi[D(x), y, z]+(-1)^{d(D) d(x)} \varphi[x, D(y), z] \\
& +(-1)^{d(D)(d(x)+d(y))} \varphi[x, y, D(z)]=[\varphi D(x), y, z] \\
& +(-1)^{d(D) d(x)}(-1)^{d(\varphi) d(x)}[x, \varphi D(y), z] \\
& +(-1)^{d(D)(d(x)+d(y))}(-1)^{d(\varphi)(d(x)+d(y))}[x, y, \varphi D(z)] \\
& =[\varphi D(x), y, z]+(-1)^{(d(D)+d(\varphi)) d(x)}[x, \varphi D(y), z] \\
& +(-1)^{(d(D)+d(\varphi))(d(x)+d(y))}(-1)^{d(\varphi)(d(x)+d(y))} \\
& +[x, y, \varphi D(z)] .
\end{aligned}
$$

Thus $\varphi D$ is a derivation.

Theorem 22. Let $T$ be a Lie supertriple system. Then for all $\varphi \in \Gamma(T)$ and $D \in \operatorname{Der}(T)$,
(1) $\operatorname{Der}(\mathfrak{g})$ is contained in the normalizer of $\Gamma(\mathfrak{g})$ in $\operatorname{End}(\mathfrak{g})$;

(2) $D \varphi$ is contained in $\Gamma(T)$ if and only if $\varphi D$ is a central derivation of $T$;

(3) $\varphi D$ is a derivation of $T$ if and only if $[D, \varphi]$ is a central derivation of $T$.

Proof. (1) For any $\varphi \in \Gamma(T), D \in \operatorname{Der}(T)$, and $x, y, z \in T$,

$$
\begin{aligned}
D \varphi & ([x, y, z])=D([\varphi(x), y, z])=[D \varphi(x), y, z] \\
& +(-1)^{(d(\varphi)+d(x)) d(D)}[\varphi(x), D(y), z] \\
& +(-1)^{(d(\varphi)+d(x)+d(y)) d(D)}[\varphi(x), y, D(z)] \\
& =[D \varphi(x), y, z]+(-1)^{(d(\varphi)+d(x)) d(D)}(-1)^{d(D) d(x)} \\
& \cdot \varphi D[(x),(y), z]+(-1)^{(d(\varphi)+d(x)+d(y)) d(D)} \\
& \cdot(-1)^{d(D)(d(x)+d(y))}[\varphi D(x), y,(z)] \\
& =[D \varphi(x), y, z]+(-1)^{d(\varphi) d(D)} \varphi D([x, y, z]) \\
& -\left[(-1)^{d(\varphi) d(D)} \varphi D(x), y, z\right] .
\end{aligned}
$$

So $\left(D \varphi-(-1)^{d(D) d(\varphi)} \varphi D\right)([x, y, z])=[(D \varphi-$ $\left.\left.(-1)^{d(D) d(\varphi)} \varphi D\right)(x), y, z\right]$; this is $[D, \varphi]=D \varphi-$ $(-1)^{d(D) d(\varphi)} \varphi D \in \Gamma(T)$. This proves (1).

(2) From Lemma 21 and (1), $D \varphi$ is an element of $\Gamma(T)$ if and only if $\varphi D \in \Gamma(T) \cap \operatorname{Der}(T)$. Thanks to Proposition 19, we get result (2).

(3) It follows from (1), Proposition 19, and Lemma 21 that (3) holds

Now, we study the relationship between the centroid of a decomposable Lie supertriple system and the centroid of its factors.

Theorem 23. Suppose that $T$ is a Lie supertriple system over $\mathbf{F}$ and $T=T_{1} \oplus T_{2}$ with $T_{1}, T_{2}$ being ideals of $T$; then

(1) $\Gamma(T) \cong \Gamma\left(T_{1}\right) \oplus \Gamma\left(T_{2}\right) \oplus C(1) \oplus C(2)$, as vector spaces, where

$$
\begin{gathered}
C(1)=\left\{\varphi \in \operatorname{Hom}\left(T_{1}, T_{2}\right) \mid \varphi\left(T_{1}\right)\right. \\
\left.\subseteq \mathrm{C}\left(T_{2}\right), \varphi\left[T_{1}, T_{1}, T_{1}\right]=0\right\}, \\
C(2)=\left\{\varphi \in \operatorname{Hom}\left(T_{2}, T_{1}\right) \mid \varphi\left(T_{2}\right)\right. \\
\left.\subseteq \mathrm{C}\left(T_{1}\right), \varphi\left[T_{2}, T_{2}, T_{2}\right]=0\right\} ;
\end{gathered}
$$

(2) $Z \operatorname{Der}(T) \cong C(1) \oplus C(2) \oplus Z \operatorname{Der}\left(T_{1}\right) \oplus Z \operatorname{Der}\left(T_{2}\right)$.

Proof. (1) Letting $\pi_{i}: T \rightarrow T_{i}$ be canonical projections for $i=1,2$, then $\pi_{1}, \pi_{2} \in \Gamma(T)$ and $\pi_{1}+\pi_{2}=\mathrm{id}_{T}$. So we have, for $\varphi \in \Gamma(T)$,

$$
\varphi=\pi_{1} \varphi \pi_{1}+\pi_{1} \varphi \pi_{2}+\pi_{2} \varphi \pi_{1}+\pi_{2} \varphi \pi_{2} .
$$


Note that $\pi_{i} \varphi \pi_{j} \in \Gamma(T)$ for $i, j=1,2$. We claim that

$$
\begin{aligned}
\Gamma(T)= & \pi_{1} \Gamma(T) \pi_{1}+\pi_{1} \Gamma(T) \pi_{2}+\pi_{2} \Gamma(T) \pi_{1} \\
& +\pi_{2} \Gamma(T) \pi_{2} .
\end{aligned}
$$

It suffices to show that $\pi_{1} \Gamma(T) \pi_{1} \cap \pi_{1} \Gamma(T) \pi_{2}=0$ (other cases are similar). For any $\varphi \in \pi_{1} \Gamma(T) \pi_{1} \cap \pi_{1} \Gamma(T) \pi_{2}$, there exist $f_{i} \in \Gamma(T), i=1,2$, such that $\varphi=\pi_{1} f_{1} \pi_{1}=\pi_{1} f_{2} \pi_{2}$. Then $\varphi(x)=\pi_{1} f_{2} \pi_{2}(x)=\pi_{1} f_{2} \pi_{2}\left(\pi_{2}(x)\right)=\pi_{1} f_{1}(0)=0$, for all $x \in T$, and so $\varphi=0$. Let $\Gamma(T)_{i j}=\pi_{i} \Gamma(T) \pi_{j}, i, j=1,2$. We now prove

$$
\begin{aligned}
& \Gamma(T)_{11} \cong \Gamma\left(T_{1}\right), \\
& \Gamma(T)_{22} \cong \Gamma\left(T_{2}\right), \\
& \Gamma(T)_{12} \cong C(2), \\
& \Gamma(T)_{21} \cong C(1) .
\end{aligned}
$$

Since $\varphi\left(T_{2}\right)=0$ for $\varphi \in \Gamma(T)_{11}$, we have $\left.\varphi\right|_{T_{1}} \in \Gamma\left(T_{1}\right)$. On the other hand, one can regard $\Gamma\left(T_{1}\right)$ as a subalgebra of $\Gamma(T)$ by extending any $\varphi_{0} \in \Gamma\left(T_{1}\right)$ on $T_{2}$ being equal to zero; that is, $\varphi_{0}\left(x_{1}\right)=\varphi_{0}\left(x_{1}\right)$ and $\varphi_{0}\left(x_{2}\right)=0$, for all $x_{1} \in T_{1}$ and $x_{2} \in T_{2}$. Then $\varphi_{0} \in \Gamma(T)$ and $\varphi_{0} \in \Gamma(T)_{11}$. Therefore $\Gamma(T)_{11} \cong \Gamma\left(T_{1}\right)$ with isomorphism $\sigma: \Gamma(T)_{11} \rightarrow \Gamma\left(T_{1}\right), \sigma(\varphi)=\left.\varphi\right|_{T_{1}}$ for all $\varphi \in \Gamma(T)_{11}$. Similarly, we have $\Gamma(T)_{22} \cong \Gamma\left(T_{2}\right)$.

Next, we prove $\Gamma(T)_{12} \cong C(2)$. If $\varphi \in \Gamma(T)_{12}$, then there exists $\varphi_{0}$ in $\Gamma(T)$ such that $\varphi=\pi_{1} \varphi_{0} \pi_{2}$. For $x_{k}=x_{k}^{1}+x_{k}^{2} \in T$, where $x_{k}^{i} \in T_{i}, i=1,2$ and $k=1,2,3$, we have

$$
\begin{aligned}
\varphi\left(\left[x_{1}, x_{2}, x_{3}\right]\right) & =\pi_{1} \varphi_{0} \pi_{2}\left(\left[x_{1}, x_{2}, x_{3}\right]\right) \\
& =\pi_{1} \varphi_{0}\left(\left[x_{1}^{2}, x_{2}^{2}, x_{3}^{2}\right]\right) \\
& =\pi_{1}\left(\left[\varphi_{0} x_{1}^{2}, x_{2}^{2}, x_{3}^{2}\right]\right)=0
\end{aligned}
$$

and $\left[\varphi\left(x_{1}\right), x_{2}, x_{3}\right]=\varphi\left(\left[x_{1}, x_{2}, x_{3}\right]\right)=0$. Then $\varphi(T) \subseteq$ $C(T)$ and $\varphi[T, T, T]=0$. It follows that $\left.\varphi\right|_{T_{2}}\left(T_{2}\right) \subseteq$ $Z\left(T_{1}\right),\left.\varphi\right|_{T_{2}}\left(\left[T_{2}, T_{2}, T_{2}\right]\right)=0$ and so $\left.\varphi\right|_{T_{2}} \in C(2)$.

Conversely, for $\varphi \in C(2)$, expanding $\varphi$ on $T$ (also denoted by $\varphi$ ) by $\varphi\left[T_{1}, T_{1}, T_{1}\right]=0$, we have $\pi_{1} \varphi \pi_{2}=\varphi$ and $\varphi \epsilon$ $\Gamma(T)_{12}$. This proves that $\Gamma(T)_{12}$ is isomorphic to $C(2)$ with the following isomorphism:

$$
\begin{aligned}
& \tau: \Gamma(T)_{12} \longrightarrow C(2), \\
& \tau(\varphi)=\left.\varphi\right|_{\Gamma(T)_{12}},
\end{aligned}
$$

for all $\varphi \in \Gamma(T)_{12}$. Similarly, we can prove $\Gamma(T)_{21} \cong C(1)$. Summarizing the above discussion we get

$$
\Gamma(T) \cong \Gamma\left(T_{1}\right) \oplus \Gamma\left(T_{2}\right) \oplus C(1) \oplus C(2) .
$$

This proves (1).

(2) Clearly, $C(1)+C(2)+\mathrm{ZDer}\left(T_{1}\right)+\mathrm{ZDer}\left(T_{2}\right) \subset \mathrm{ZDer}(T)$. Letting $\pi_{i}: T \rightarrow T_{i}$ be canonical projections for $i=1,2$, then $\pi_{1}, \pi_{2} \in \Gamma(T)$ and $\pi_{1}+\pi_{2}=\mathrm{id}_{T}$. So we have, for $\varphi \in \operatorname{ZDer}(T), \varphi=\pi_{1} \varphi \pi_{1}+\pi_{1} \varphi \pi_{2}+\pi_{2} \varphi \pi_{1}+\pi_{2} \varphi \pi_{2}$. Note that $\pi_{i} \varphi \pi_{j} \in \operatorname{ZDer}(T)$ for $i, j=1,2$. We claim that
$\operatorname{ZDer}(T)=\pi_{1} \operatorname{ZDer}(T) \pi_{1}+\pi_{1} \operatorname{ZDer}(T) \pi_{2}+\pi_{2} \mathrm{ZDer}(T) \pi_{1}+$ $\pi_{2} \mathrm{ZDer}(T) \pi_{2}$. Let $\mathrm{ZDer}(T)_{i j}=\pi_{i} \mathrm{ZDer}(T) \pi_{j}, i, j=1,2$; we now have $\mathrm{ZDer}(T)_{11} \cong \mathrm{ZDer}\left(T_{1}\right), \mathrm{ZDer}(T)_{22} \cong \mathrm{ZDer}\left(T_{2}\right)$, $\mathrm{ZDer}(T)_{12} \cong C(2)$, and $\mathrm{ZDer}(T)_{21} \cong C(1)$. In the same way as (1), we get (2).

A generalized version of the above theorem is stated below without proof.

Theorem 24. Suppose that $T$ is a Lie supertriple system over $\mathbf{F}$ with a decomposition of ideals $T=T_{1} \oplus T_{2} \oplus \cdots \oplus T_{m}$. Then we have

(1) $\Gamma(T) \cong \Gamma\left(T_{1}\right) \oplus \cdots \oplus \Gamma\left(T_{m}\right) \oplus\left(\oplus_{1 \leq i \neq j \leq m} C_{i j}\right)$ as vector spaces, where $C_{i j}=\left\{\varphi \in \operatorname{Hom}\left(T_{i}, T_{j}\right) \mid \varphi\left(T_{i}\right) \subseteq\right.$ $\mathrm{C}\left(T_{j}\right), \varphi\left[T_{i}, T_{i}, T_{i}\right]=0$, for $\left.1 \leq i \neq j \leq m\right\} ;$

(2) $Z \operatorname{Der}(T) \cong Z \operatorname{Der}\left(T_{1}\right) \oplus \cdots \oplus Z \operatorname{Der}\left(T_{m}\right) \oplus\left(\oplus_{1 \leq i \neq j \leq m} C_{i j}\right)$.

\section{Centroid of Tensor Product}

Benkart and Neher investigated the centroid of the tensor product of associative algebras and Lie algebras in [8]. In this section we discuss the centroid of the tensor product of a Lie supertriple system and a unital commutative associative algebra. Furthermore, we completely determine the centroid of the tensor product $T \otimes R$ of a simple Lie supertriple system $T$ and a polynomial ring $R$.

Definition 25. Let $A$ be an associative algebra over a field $\mathbf{F}$. The centroid of $A$ is the space of $\mathbf{F}$-linear transformations on $A$ given by $\Gamma(A)=\left\{\chi \in \operatorname{End}_{\mathrm{F}}(A) \mid \chi(a b)=a \chi(b)=\right.$ $\chi(a) b, \forall a, b \in A\}$.

Proposition 26. Let $T$ be a Lie supertriple system over $\mathbf{F}$ and $A$ a unital commutative associative algebra over $\mathbf{F}$. Then there exists a unique Lie supertriple system structure on $T \otimes A$ satisfying $\left[x \otimes a_{1}, y \otimes a_{2}, z \otimes a_{3}\right]=[x, y, z] \otimes a_{1} a_{2} a_{3}, \forall x, y, z \in T$, $a_{i} \in A, i=1,2,3$.

Proof. For $u, v, x, y, z \in T, a_{i} \in A, i=1,2,3,4,5$, we have

$$
\begin{aligned}
& d\left(\left[x \otimes a_{1}, y \otimes a_{2}, z \otimes a_{3}\right]\right) \equiv d(x)+d(y)+d(z)(\bmod 2), \\
& {\left[x \otimes a_{1}, y \otimes a_{2}, z \otimes a_{3}\right]=[x, y, z] \otimes a_{1} a_{2} a_{3}} \\
& \quad=-(-1)^{d(x) d(y)}[y, x, z] \otimes a_{2} a_{1} a_{3}=-(-1)^{d(x) d(y)} \\
& \quad \cdot\left[y \otimes a_{2}, x \otimes a_{1}, z \otimes a_{3}\right] \\
& \quad=-(-1)^{d\left(x \otimes a_{1}\right) d\left(y \otimes a_{2}\right)}\left[y \otimes a_{2}, x \otimes a_{1}, z \otimes a_{3}\right] \\
& (-1)^{d\left(x \otimes a_{1}\right) d\left(z \otimes a_{3}\right)}\left[x \otimes a_{1}, y \otimes a_{2}, z \otimes a_{3}\right] \\
& \quad+(-1)^{d\left(x \otimes a_{1}\right) d\left(y \otimes a_{2}\right)}\left[y \otimes a_{2}, z \otimes a_{3}, x \otimes a_{1}\right] \\
& \quad+(-1)^{d\left(y \otimes a_{2}\right) d\left(z \otimes a_{3}\right)}\left[z \otimes a_{3}, x \otimes a_{1}, y \otimes a_{2}\right] \\
& \quad=(-1)^{d\left(x \otimes a_{1}\right) d\left(z \otimes a_{3}\right)}[x, y, z] \otimes a_{1} a_{2} a_{3} \\
& \quad+(-1)^{d\left(x \otimes a_{1}\right) d\left(y \otimes a_{2}\right)}[y, z, x] \otimes a_{2} a_{3} a_{1} \\
& \quad+(-1)^{d\left(y \otimes a_{2}\right) d\left(z \otimes a_{3}\right)}[z, x, y] \otimes a_{3} a_{1} a_{2}
\end{aligned}
$$




$$
\begin{aligned}
&=\left((-1)^{d(x) d(z)}[x, y, z]+(-1)^{d(x) d(y)}[y, z, x]\right. \\
&\left.+(-1)^{d(y) d(z)}[z, x, y]\right) \otimes a_{1} a_{2} a_{3}=0 \otimes a_{1} a_{2} a_{3}=0, \\
& {\left[u \otimes a_{1}, v \otimes a_{2},\left[x \otimes a_{3}, y \otimes a_{4}, z \otimes a_{5}\right]\right] } \\
& \quad=[u, v,[x, y, z]] \otimes a_{1} a_{2} a_{3} a_{4} a_{5}=([[u, v, x], y, z] \\
&+(-1)^{(d(u)+d(v)) d(x)}[x,[u, v, y], z] \\
&\left.+(-1)^{(d(u)+d(v))(d(x)+d(y))}[x, y,[u, v, z]]\right) \\
& \otimes a_{1} a_{2} a_{3} a_{4} a_{5}=[[u, v, x], y, z] \otimes a_{1} a_{2} a_{3} a_{4} a_{5} \\
&+(-1)^{(d(u)+d(v)) d(x)}[x,[u, v, y], z] \otimes a_{1} a_{2} a_{3} a_{4} a_{5} \\
&=\left[\left[u \otimes a_{1}, v \otimes a_{2}, x \otimes a_{3}\right], y \otimes a_{4}, z \otimes a_{5}\right] \\
&+(-1)^{\left(d\left(u \otimes a_{1}\right)+d\left(v \otimes a_{2}\right)\right) d\left(x \otimes a_{3}\right)}[x \\
&\left.\otimes a_{3},\left[u \otimes a_{1}, v \otimes a_{2}, y \otimes a_{4}\right], z \otimes a_{5}\right] \\
&+(-1)^{\left(d\left(u \otimes a_{1}\right)+d\left(v \otimes a_{2}\right)\right)\left(d\left(x \otimes a_{3}\right)+d\left(y \otimes a_{4}\right)\right)}\left[x \otimes a_{3}, y\right. \\
&\left.\otimes a_{4},\left[u \otimes a_{1}, v \otimes a_{2}, z \otimes a_{5}\right]\right] .
\end{aligned}
$$

Thus $T \otimes A$ is a Lie supertriple system.

It is easy to show that if $T$ is perfect, then $T \otimes A$ is perfect too. Also, for $f \in \operatorname{End}_{\mathrm{F}}(T)$ and $g \in \operatorname{End}_{\mathrm{F}}(A)$ there exists a unique map $f \widetilde{\otimes} g \in \operatorname{End}_{\mathrm{F}}(T \otimes A)$ such that

$$
(f \widetilde{\otimes} g)(x \otimes a)=f(x) \otimes g(a), \quad \forall x \in T, a \in A .
$$

The map should not be confused with the element $f \otimes g$ of the tensor product $\operatorname{End}_{\mathbf{F}}(T) \otimes \operatorname{End}_{\mathbf{F}}(A)$. Of course, we have a canonical map

$$
\begin{aligned}
\omega: \operatorname{End}_{\mathbf{F}}(T) \otimes \operatorname{End}_{\mathbf{F}}(A) \longrightarrow \operatorname{End}_{\mathbf{F}}(T \otimes A), & \\
f \otimes g & \longmapsto f \widetilde{\otimes} g .
\end{aligned}
$$

It is easy to see that if $\phi \in \Gamma(T)$ and $\psi \in \Gamma(A)$, then $\phi \widetilde{\otimes} \psi \epsilon$ $\Gamma(T \otimes A)$. Hence $\Gamma(T) \widetilde{\otimes} \Gamma(A) \subseteq \Gamma(T \otimes A)$, where $\Gamma(T) \widetilde{\otimes} \Gamma(A)$ is the F-span of all endomorphisms $\phi \widetilde{\otimes} \psi$.

Definition 27. The transformation $\phi \in \Gamma(T \otimes A)$ has finite $T$ image if, for any $a \in A$, there exist finitely many $a_{1}, \ldots, a_{n} \in A$ such that

$$
\phi(T \otimes \mathbf{F} a) \subseteq T \otimes \mathbf{F} a_{1}+\cdots+T \otimes \mathbf{F} a_{n} .
$$

It is easy to see that $\Gamma(T) \widetilde{\otimes} \Gamma(A) \subseteq\{\phi \in \Gamma(T \otimes A) \mid \phi$ has finite $T$-image $\}$. In addition, if $T$ is perfect, $\phi \in \Gamma(T \otimes A)$ has finite $T$-image as soon as $\phi(T \otimes 1) \subseteq T \otimes \mathbf{F} a_{1}+\cdots+T \otimes \mathbf{F} a_{n}$ for suitable $a_{i} \in A$.

Lemma 28. Let $T$ be a Lie supertriple system over $\mathbf{F}$ and $A$ a unital commutative associative $\mathbf{F}$-algebra. Let $\left\{a_{r}\right\}_{r \in \Re}$ be a basis of $A$ and $\phi \in \Gamma(T \otimes A)$. One defines $\phi_{r} \in \operatorname{End}_{\mathrm{F}}(T)$ by

$$
\phi(x \otimes 1)=\sum_{r \in \Re} \phi_{r}(x) \otimes a_{r} .
$$

Then all $\phi_{r} \in \Gamma(T)$.
Proof. For $x_{1}, x_{2}, x_{3} \in T$, we have $\phi\left(\left[x_{1}, x_{2}, x_{3}\right] \otimes 1\right)=$ $\sum_{r \in \mathfrak{R}} \phi_{r}\left(\left[x_{1}, x_{2}, x_{3}\right]\right) \otimes a_{r}$ and

$$
\begin{aligned}
\phi\left(\left[x_{1}, x_{2}, x_{3}\right] \otimes 1\right) & =\phi\left(\left[x_{1} \otimes 1, x_{2} \otimes 1, x_{3} \otimes 1\right]\right) \\
& =\left[\phi\left(x_{1} \otimes 1\right), x_{2} \otimes 1, x_{3} \otimes 1\right] \\
& =\sum_{r \in \Re}\left[\phi_{r}\left(x_{1}\right), x_{2}, x_{3}\right] \otimes a_{r} .
\end{aligned}
$$

Hence $\phi_{r}\left(\left[x_{1}, x_{2}, x_{3}\right]\right)=\left[\phi_{r}\left(x_{1}\right), x_{2}, x_{3}\right]=\left[x_{1}, \phi_{r}\left(x_{2}\right), x_{3}\right]=$ $\left[x_{1}, x_{2}, \phi_{r}\left(x_{3}\right)\right]$ for all $x_{i} \in T$, so all $\phi_{r} \in \Gamma(T)$.

Proposition 29. Let $T$ be a perfect Lie supertriple system over $\mathbf{F}$ and $A$ a unital commutative associative $\mathbf{F}$-algebra. Then,

(1) if $T$ is finite generated as an InnDer(T)-module (or as a $\Gamma(T)$-module) or $T$ is central, every $\phi \in \Gamma(T \otimes A)$ has finite T-image;

(2) if the map $\omega$ in Definition 25 is injective, $\Gamma(T) \widetilde{\otimes} \Gamma(A)=$ $\{\phi \in \Gamma(T \otimes A) \mid \phi$ has finite $T$-image $\}$.

Proof. (1) Set $I=\operatorname{InnDer}(T)$. Since $A$ is unital, $I \widetilde{\otimes}$ id $\subseteq$ $\operatorname{InnDer}(T \otimes A)$. Since $T$ is finite generated as an $\operatorname{InnDer}(T)$ module, we can suppose $T=I x_{1}+\cdots+I x_{n}=I_{1} x_{1}+\cdots+I_{n} x_{n}$ for $x_{1}, \ldots, x_{n} \in T, I_{1}, \ldots, I_{n} \in I$. Fix $\phi \in \Gamma(T \otimes A)$ and $a \in A$. There exist finite families $\left\{x_{i j}\right\} \subseteq T$ and $\left\{a_{i j}\right\} \subseteq A$ such that $\phi\left(x_{i} \otimes a\right)=\sum_{j} x_{i j} \otimes a_{i j}$ for $1 \leq i \leq n$. Hence

$$
\begin{aligned}
\phi(T \otimes a) & =\sum_{i} \phi\left(I_{i} \widetilde{\otimes} \mathrm{id}\right)\left(x_{i} \otimes a\right) \\
& =(-1)^{d(\phi) d\left(I_{i} \widetilde{\otimes i d}\right)} \sum_{i}\left(I_{i} \widetilde{\otimes} \mathrm{id}\right) \phi\left(x_{i} \otimes a\right) \\
& =(-1)^{d(\phi) d\left(I_{i} \widetilde{\otimes i d}\right)} \sum_{i, j}\left(I_{i} \widetilde{\otimes} \mathrm{id}\right)\left(x_{i j} \otimes a_{i j}\right) \\
& \subseteq \sum_{i, j} T \otimes a_{i j} .
\end{aligned}
$$

Since $T \otimes A$ is perfect, the centroid $\Gamma(T \otimes A)$ is supercommutative. Replacing $I$ by $\Gamma(T)$ we can use the same argument as above to show that every $\phi \in \Gamma(T \otimes A)$ has finite $T$-image if $T$ is a finitely generated $\Gamma(T)$-module.

Now, suppose $\Gamma(T)=$ Fid and $T$ is a torsion-free $\mathbf{F}$ module. Then there exist scalars $k_{r} \in \mathbf{F}$ such that $\phi_{r}=k_{r}$ id. Hence $\phi(x \otimes 1)=\sum_{r \in \Re} k_{r} x \otimes a_{r}$. Fix $x \in T$; then almost all $k_{r} x=0$, so almost all $k_{r}=0$, which in turn implies that $\phi$ has finite $T$-image.

(2) In the discussion above, we get $\Gamma(T) \widetilde{\otimes} \Gamma(A) \subseteq\{\phi \epsilon$ $\Gamma(T \otimes A) \mid \phi$ has finite $T$-image $\}$. So it suffices to prove $\Gamma(T) \widetilde{\otimes} \Gamma(A) \supseteq\{\phi \in \Gamma(T \otimes A) \mid \phi$ has finite $T$-image $\}$. We suppose that $\phi \in \Gamma(T \otimes A)$ has finite $T$-image; then there exists a finite subset $\mathfrak{J} \subseteq \mathfrak{R}$ such that $\phi(x \otimes 1)=\sum_{r \in \mathfrak{I}} \phi_{r}(x) \otimes a_{r}$. For $x_{1}, x_{2}, x_{3} \in T$ and $a \in A$, we get

$$
\begin{aligned}
\phi\left(\left[x_{1}, x_{2}, x_{3}\right] \otimes a\right) & =\phi\left(\left[x_{1} \otimes 1, x_{2} \otimes 1, x_{3} \otimes a\right]\right) \\
& =\left[\phi\left(x_{1} \otimes 1\right), x_{2} \otimes 1, x_{3} \otimes a\right]
\end{aligned}
$$




$$
\begin{aligned}
& =\sum_{r \in \mathfrak{I}}\left[\phi_{r}\left(x_{1}\right), x_{2}, x_{3}\right] \otimes a_{r} a \\
& =\sum_{r \in \mathfrak{I}} \phi_{r}\left(\left[x_{1}, x_{2}, x_{3}\right]\right) \otimes a_{r} a .
\end{aligned}
$$

Since $T$ is perfect, we can get $\phi=\sum_{r \in \mathfrak{I}} \phi_{r} \widetilde{\otimes} \lambda_{r}$, where $\lambda_{r}$ is the left multiplication in $A$ by $a_{r}$. Let $\left\{\psi_{s} \mid s \in \mathbb{S}\right\}$ be an F-basis of $\Gamma(T)$. Then there exist a finite subset $\mathfrak{T} \subseteq \mathbb{S}$ and scalars $k_{r s} \in \mathbf{F}(r \in \mathfrak{J}, s \in \mathfrak{I})$ such that $\phi_{r}=\sum_{s \in \mathfrak{T}} k_{r s} \psi_{s}$. We then get $\phi=\sum_{r \in \mathfrak{T}} \sum_{s \in \mathfrak{T}} k_{r s} \psi_{s} \widetilde{\otimes} \lambda_{r}=\sum_{s \in \mathfrak{T}} \psi_{s} \widetilde{\otimes} \phi_{s}$ for $\phi_{s}=\sum_{r \in \mathfrak{T}} k_{r s} \lambda_{r}$. Now we show that $\phi_{s} \in \Gamma(A)$. For any $x_{1}, x_{2}, x_{3} \in T, a_{1}, a_{2} \in$ $A$, we have

$$
\begin{aligned}
\sum_{s \in \mathfrak{I}} \psi_{s} & \left(\left[x_{1}, x_{2}, x_{3}\right]\right) \otimes \phi_{s}\left(a_{1} a_{2}\right) \\
& =\phi\left(\left[x_{1}, x_{2}, x_{3}\right] \otimes a_{1} a_{2}\right) \\
& =\left[\phi\left(x_{1} \otimes a_{1}\right), x_{2} \otimes a_{2}, x_{3} \otimes 1\right] \\
& =\left[\sum_{s \in \mathfrak{I}} \psi_{s}\left(x_{1}\right) \otimes \phi_{s}\left(a_{1}\right), x_{2} \otimes a_{2}, x_{3} \otimes 1\right] \\
& =\sum_{s \in \mathfrak{T}}\left[\psi_{s}\left(x_{1}\right), x_{2}, x_{3}\right] \otimes \phi_{s}\left(a_{1}\right) a_{2} \\
& =\sum_{s \in \mathfrak{I}} \psi_{s}\left(\left[x_{1}, x_{2}, x_{3}\right]\right) \otimes \phi_{s}\left(a_{1}\right) a_{2} .
\end{aligned}
$$

Because $T^{(1)}=T$, we can get $\sum_{s \in \mathfrak{I}} \psi_{s}(x) \otimes\left(\phi_{s}\left(a_{1} a\right)-\phi_{s}\left(a_{1}\right) a\right)=$ $0, \forall x \in T$, and $a_{1}, a_{2} \in A$; it follows that $\sum_{s \in \mathfrak{I}} \psi_{s} \widetilde{\otimes} \mu_{s}=0$ where $\mu_{s} \in \operatorname{End}_{\mathrm{F}}(A)$ is defined by $\mu_{s}\left(a_{2}\right)=\phi_{s}\left(a_{1} a_{2}\right)-\phi_{s}\left(a_{1}\right) a_{2}$, $\forall a_{2} \in A$. Since $\omega$ is injective, we also have $\sum_{s \in \mathfrak{I}} \psi_{s} \otimes \mu_{s}=0$. So by the linear independence of the $\psi_{s}$, we get that $\mu_{s}=0$. Then $\psi_{s} \in \Gamma(A)$. Hence $\phi \in \Gamma(T) \widetilde{\otimes} \Gamma(A)$.

Next we will determine the centroid of the tensor product of a simple Lie supertriple system and a polynomial ring.

Let $T$ be a Lie supertriple system and $R=\mathbf{F}\left[x_{1}, \ldots, x_{n}\right]$. Defining the multiplication by $[x \otimes p, y \otimes q, z \otimes r]=[x, y, z] \otimes$ $p q r$, we can make $\widetilde{T}=T \otimes R$ into a Lie supertriple system.

Now we write out a basis of $\Gamma(T)$ in the following manner. First, let $E$ denote the set of central derivations and choose a basis $\left\{e_{k}\right\}$ for $E$. Second, let $\left\{\psi_{j}\right\}$ be a maximal subset of $\Gamma(T)$ with the restrictions $\left\{\left.\psi_{j}\right|_{[T, T, T]}\right\}$ being linearly independent and $\Psi$ the space spanned by $\psi_{j}$. Then $\Gamma(T)=\Psi+E ;\left\{e_{k}\right\}$ and $\left\{\psi_{j}\right\}$ form a basis of $\Gamma(T)$.

Lemma 30. One has $\Gamma(\widetilde{T}) \supseteq \Psi \otimes R+E \otimes \operatorname{End}_{\mathbf{F}}(R)$.

Proof. Let $\psi \in \Gamma(T), m \in R$. Then we have $(\psi \otimes m)([x \otimes p, y \otimes$ $q, z \otimes r])=(\psi \otimes m)([x, y, z] \otimes p q r)=\psi([x, y, z]) \otimes m p q r=$ $[\psi(x), y, z] \otimes m p q r$ and $[(\psi \otimes m)(x \otimes p), y \otimes q, z \otimes r]=[\psi(x) \otimes$ $m p, y \otimes q, z \otimes r]=[\psi(x), y, z] \otimes m p q r$. Thus $\psi \otimes m \in \Gamma(\widetilde{T})$.

For any $d \in E$ and $f \in \operatorname{End}_{\mathrm{F}}(R), d \otimes f \in \Gamma(\widetilde{T})$ since $(d \otimes f)([x \otimes p, y \otimes q, z \otimes r])=d([x, y, z]) \otimes f(p q r)=0$ and $[(d \otimes f)(x \otimes p), y \otimes q, z \otimes r]=[d(x) \otimes f(p), y \otimes q, z \otimes r]=$ $[d(x), y, z] \otimes f(p) q r=0$. Hence $\Psi \otimes R+E \otimes \operatorname{End}_{\mathbf{F}}(R) \subseteq$ $\Gamma(\widetilde{T})$.
Theorem 31. Letting $T$ be a simple Lie supertriple system, $R=$ $\mathbf{F}\left[x_{1}, \ldots, x_{n}\right]$ and $\widetilde{T}=T \otimes R$. Then $\Gamma(\widetilde{T})_{\overline{0}}=\Gamma(T)_{\overline{0}} \otimes R$.

Proof. From Lemma 30, we get $\Gamma(T) \otimes R \subseteq \Gamma(\widetilde{T})$, so $\Gamma(T)_{\overline{0}} \otimes R \subseteq$ $\Gamma(\widetilde{T})_{\overline{0}}$. Now we prove the opposite inclusion.

Let $\left\{m_{i}\right\}$ be a basis for $R$. Let $\psi \in \Gamma(\widetilde{T})_{\overline{0}}, p \in R, x, y, z \in T$ and $\eta_{i}(-, p)$ be suitable maps in $\operatorname{End}_{\mathbf{F}}(T)$ such that we may write

$$
\psi(x \otimes p)=\sum_{i} \eta_{i}(x, p) \otimes m_{i}
$$

Then

$$
\begin{aligned}
\psi( & {[x \otimes p, y \otimes 1, z \otimes 1]) } \\
& =[\psi(x \otimes p), y \otimes 1, z \otimes 1] \\
& =\left[\sum_{i} \eta_{i}(x, p) \otimes m_{i}, y \otimes 1, z \otimes 1\right] \\
& =\sum_{i}\left[\eta_{i}(x, p), y, z\right] \otimes m_{i},
\end{aligned}
$$

while

$$
\psi([x, y, z] \otimes p)=\sum_{i} \eta_{i}([x, y, z], p) \otimes m_{i} .
$$

So, for each $i$ and $p$, we have $\eta_{i}(p,[x, y, z])=\left[\eta_{i}(x, p), y, z\right]$, $\forall x, y, z \in T$. Hence $\eta_{i}(-, p) \in \Gamma(T)$. But $\Gamma(T)=$ Fid. Therefore, $\eta_{i}(x, p)=\lambda_{i}(p) x, \forall x \in T$, for suitable scalars $\lambda_{i}(p)$.

Thus, now we may write $\psi(x \otimes p)=\sum_{i} \lambda_{i}(p) x \otimes m_{i}$. Since the right hand side is in $T \otimes R$, for each $p$, we get $\lambda_{i}(p)=0$ for all except a finite number of $i$. That is, $\psi(x \otimes p)=\sum_{i}^{n} x \otimes$ $\lambda_{i}(p) m_{i}$. Then the map $\rho: p \mapsto \sum_{i}^{n} \lambda_{i}(p) m_{i}$ is well defined. Hence, we have $\psi(x \otimes p)=x \otimes \rho(p)$. Then we get

$$
\begin{aligned}
{[\psi} & (x \otimes p), y \otimes 1, z \otimes 1] \\
& =[x \otimes \rho(p), y \otimes 1, z \otimes 1]=[x, y, z] \otimes \rho(p) \\
& =(-1)^{d(\psi) d(x \otimes p)}[x \otimes p, \psi(y \otimes 1), z \otimes 1] \\
& =(-1)^{d(\psi) d(x \otimes p)}[x \otimes p, y \otimes \rho(1), z \otimes 1] \\
& =[x, y, z] \otimes(-1)^{d(\psi) d(x \otimes p)} p \rho(1) \\
& =[x, y, z] \otimes p \rho(1) .
\end{aligned}
$$

Choose any triple $x, y, z \in T$ such that $[x, y, z] \neq 0$. Then we can conclude that $\rho(p)=p \rho(1), \forall p \in R$. So $\rho$ is determined by its action on 1 . Therefore, $\rho \in R$. Thus, $\psi(x \otimes p)=($ id $\otimes$ $\rho)(x \otimes p), \forall p \in R, x \in T$. That is, $\psi \in \operatorname{id} \otimes R \subseteq \Gamma(T)_{\overline{0}} \otimes R$. So we conclude that $\Gamma(\widetilde{T})_{\overline{0}}=\Gamma(T)_{\overline{0}} \otimes R$.

\section{Conflict of Interests}

The authors declare that there is no conflict of interests regarding the publication of this paper. 


\section{Acknowledgments}

The authors would like to thank the referee for valuable comments and suggestions on this paper. This research was supported by NNSF of China (nos. 11171055 and 11471090) and the Fundamental Research Funds for the Central University (no. 14ZZ2221).

\section{References}

[1] N. Jacobson, "Lie and Jordan triple systems," American Journal of Mathematics, vol. 71, pp. 149-170, 1949.

[2] S. Okubo, "Parastatistics as Lie-supertriple systems," Journal of Mathematical Physics, vol. 35, no. 6, pp. 2785-2803, 1994.

[3] I. Bars and M. Günaydin, "Construction of Lie algebras and Lie superalgebras from ternary algebras," Journal of Mathematical Physics, vol. 20, no. 9, pp. 1977-1993, 1978.

[4] N. Kamiya and S. Okubo, "On $\delta$-lie supertriple systems associated with $(\epsilon, \delta)$-Freudenthal-Kantor supertriple systems," Proceedings of the Edinburgh Mathematical Society, vol. 43, pp. 243-260, 2000.

[5] Y. Ma, L. Y. Chen, and D. Liu, "On generalized derivations of lie supertriple systems," Acta Mathematica Sinica, vol. 56, no. 6, pp. 961-970, 2013 (Chinese).

[6] S. Okubo, "Yang-Baxter equation and triple product systems," in Proceedings of the 6th International Symposium on Symmetries in Science, pp. 561-568, Bregenz, Austria, 1992.

[7] S. Okubo and N. Kamiya, "Quasi-classical Lie superalgebras and Lie supertriple systems," Communications in Algebra, vol. 30, no. 8, pp. 3825-3850, 2002.

[8] G. Benkart and E. Neher, "The centroid of extended affine and root graded Lie algebras," Journal of Pure and Applied Algebra, vol. 205, no. 1, pp. 117-145, 2006.

[9] D. J. Melville, "Centroids of nilpotent Lie algebras," Communications in Algebra, vol. 20, no. 12, pp. 3649-3682, 1992.

[10] P. Benito, C. Draper, and A. Elduque, "On some algebras related to simple lie triple systems," Journal of Algebra, vol. 219, no. 1, pp. 234-254, 1999.

[11] R. Bai, H. An, and Z. Li, "Centroid structures of n-Lie algebras," Linear Algebra and Its Applications, vol. 430, no. 1, pp. 229-240, 2009.

[12] J. Lie and Y. Wang, "Centroid of Lie triple systems," Acta Scientiarum Naturalium Universitatis Nankaiensis (Science Edition), vol. 43, pp. 98-104, 2010.

[13] X. Liu and L. Chen, "The centroid of a Lie triple algebra," Abstract and Applied Analysis, vol. 2013, Article ID 404219, 9 pages, 2013.

[14] J. J. Rotman, A First Course in Abstract Algebra, Prentice Hall, Upper Saddle River, NJ, USA, 1996.

[15] N. Jacobson, Lectures in Abstract Algebra. I, D. Van Nostrand, London, UK, 1951.

[16] V. G. Kac, "Lie superalgebras," Advances in Mathematics, vol. 26, no. 1, pp. 8-96, 1977. 


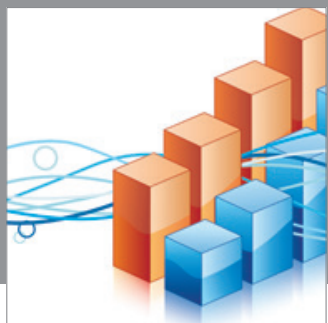

Advances in

Operations Research

mansans

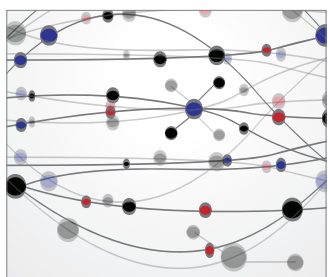

The Scientific World Journal
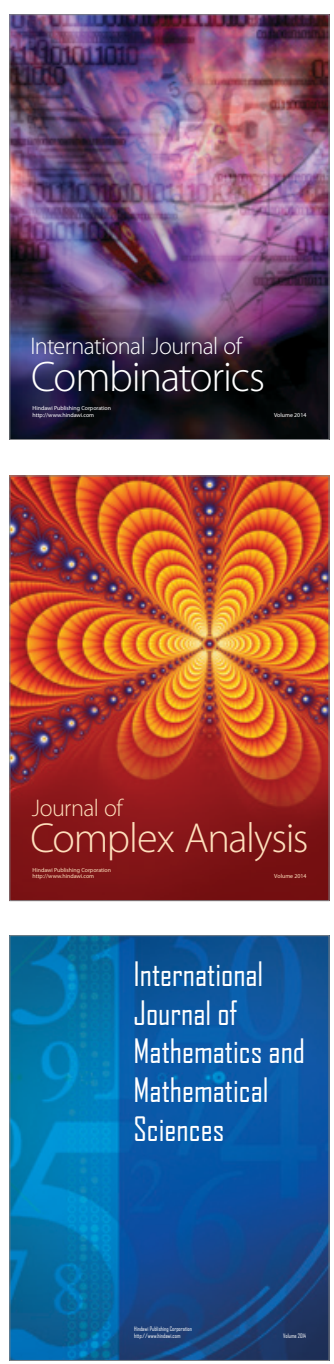
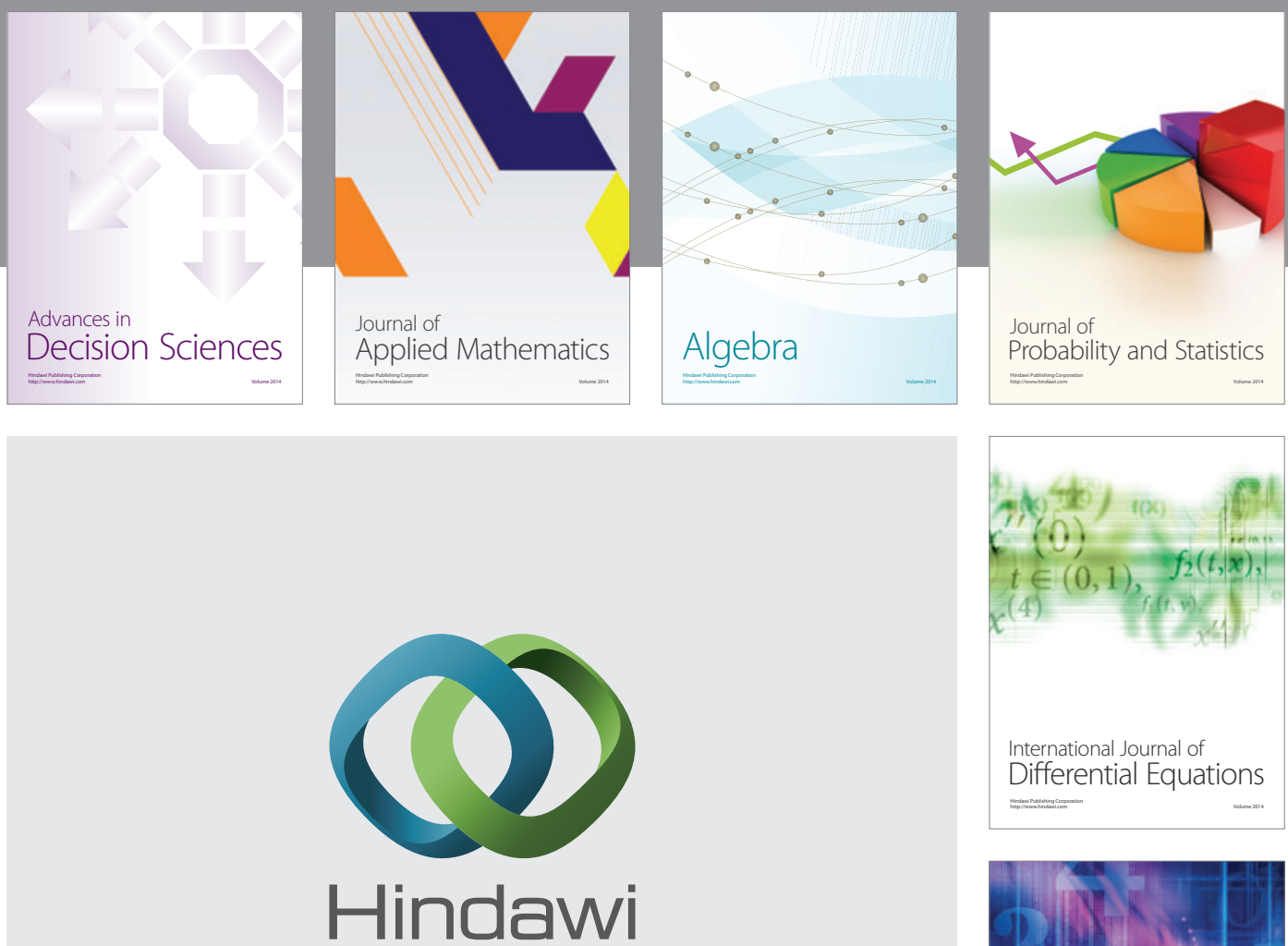

Submit your manuscripts at http://www.hindawi.com
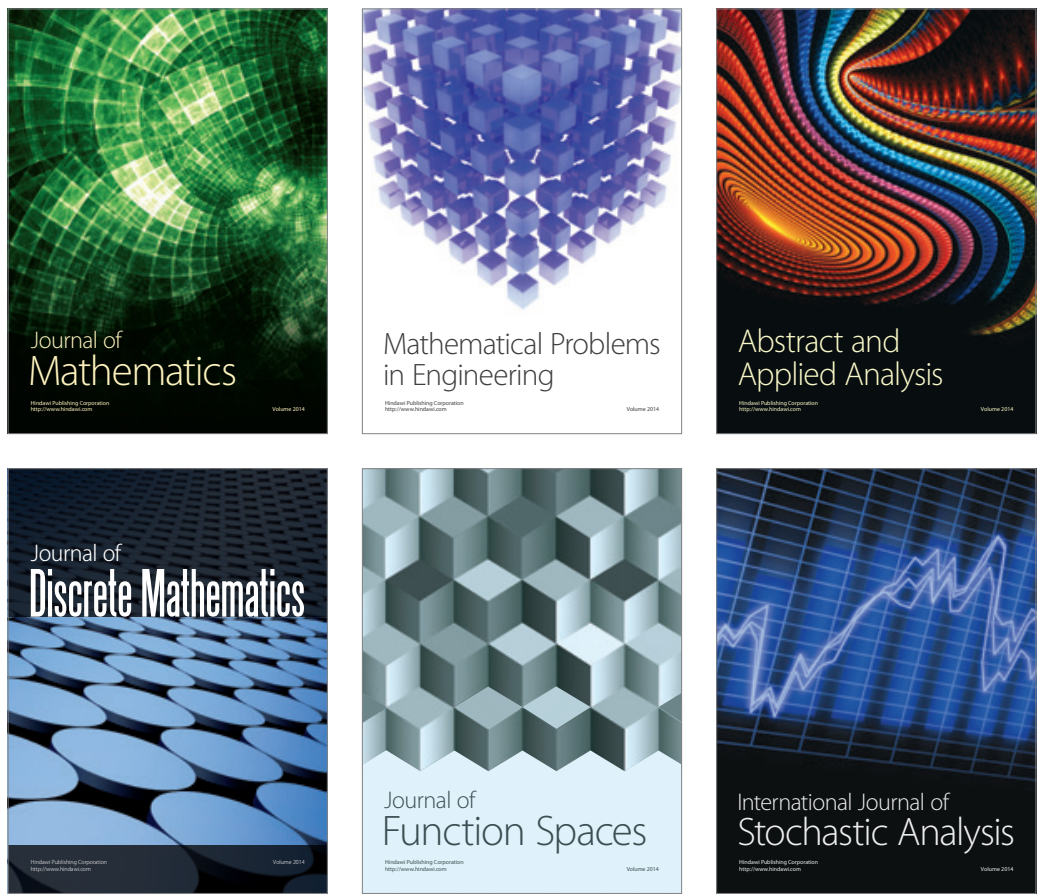

Journal of

Function Spaces

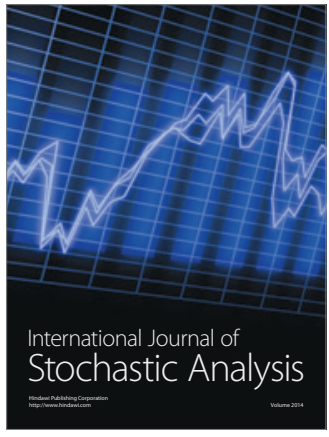

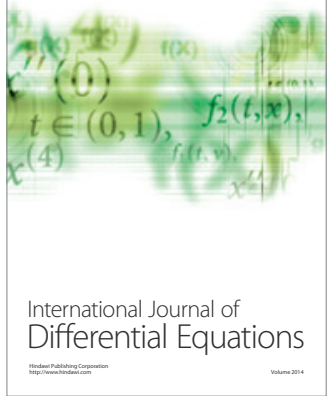
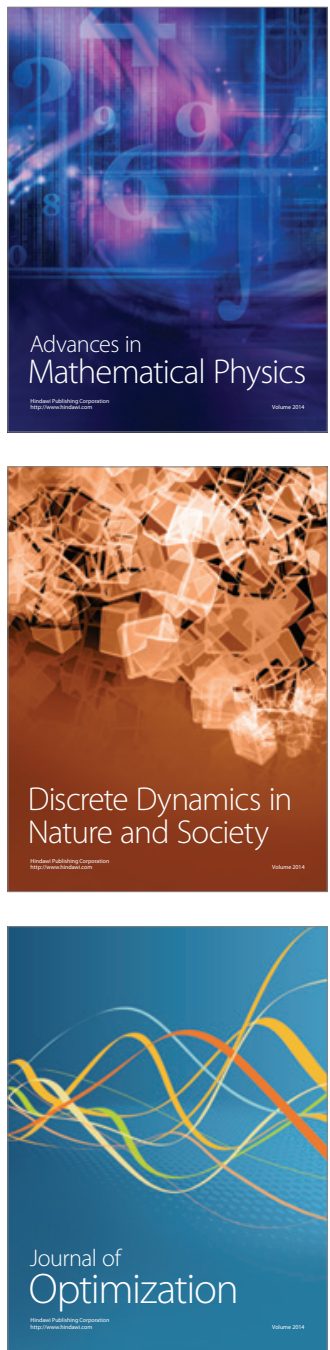